Rapport - Société canadienne d'histoire de l'Église catholique

\title{
Le Troisième Centenaire de la Congrégation de la Haute-Ville de Québec
}

\section{Adrien Pouliot}

Volume 24, 1956-1957

URI : https://id.erudit.org/iderudit/1007436ar

DOI : https://doi.org/10.7202/1007436ar

Aller au sommaire du numéro

Éditeur(s)

La Société canadienne d'histoire de l'Église catholique

ISSN

0318-6148 (imprimé)

1927-7075 (numérique)

Découvrir la revue

Citer cet article

Pouliot, A. (1956). Le Troisième Centenaire de la Congrégation de la Haute-Ville de Québec. Rapport - Société canadienne d'histoire de l'Église catholique, 24,

103-121. https://doi.org/10.7202/1007436ar

Tous droits réservés @ La Société canadienne d'histoire de l'Église catholique, 1958
Ce document est protégé par la loi sur le droit d'auteur. L’utilisation des services d'Érudit (y compris la reproduction) est assujettie à sa politique d'utilisation que vous pouvez consulter en ligne.

https://apropos.erudit.org/fr/usagers/politique-dutilisation/ 


\section{Le Troisième Centenaire de la Congrégation de la Haute-Ville de Québec}

Quelle plus belle journée pour parler, ici à Montréal, des congrégations mariales canadiennes, que cette fête du très saint Nom de Marie (12 septembre), fête patronale de Ville-Marie, fête titulaire d'un diocèse dont l'éminent cardinal-archevêque leur porte un si paternel et si efficace attachement !

Il y a actuellement au Canada, réparties dans une soixantaine de diocèses, environ deux mille congrégations mariales reconnues comme telles par les deux secrétariats nationaux de Montréal et de Toronto : soit, en chiffres ronds, douze cents dans le secteur français et huit cents dans le secteur anglais. Sur les douze cents du secteur français, mille sont féminines, aux deux tiers paroissiales, et deux cents sont masculines, se partageant également entre les paroisses et les institutions scolaires. Il y a de plus six congrégations de prêtres ou de grands séminaristes et cinq congrégations mixtes, groupant ensemble hommes et femmes, jeunes gens et jeunes filles.

Ces chiffres sont à la fois supérieurs et inférieurs à la réalité : supérieurs, parce qu'un trop grand nombre de ces congrégations n'ont pas encore fait passer dans leur vie les réformes imposées par la Constitution apostolique Bis saculari, du 27 septembre 1948; inférieurs, parce que plusieurs congrégations nouvelles et actives n'ont pas encore terminé la période d'épreuve maintenant requise pour l'agrégation.

Quoi qu'il en soit, un fait s'impose, cette année, à notre réflexion : toutes ces congrégations, d'hier ou d'aujourd'hui, rénovées ou routinières, lampes ardentes ou mèches fumantes, doivent leur origine à une étincelle initiale. Cette étincelle initiale qui, après avoir allumé d'autres feux, brûle encore du sien, ce fut la Congrégation des Hommes de la HauteVille de Québec, fondée le 14 février 1657.

* Rappelez-vous les jours anciens », conseille l'Apôtre (Epître aux Hébreux, $\mathrm{X}, 32$ ). Il y a toujours avantage à remonter aux origines. Dans le cas des congrégations mariales, le désir qu'a le Saint-Père de les voir partout refleurir nous en fait un devoir. Mon intention n'est pas - et ne peut pas être - de vous raconter en trente minutes les trois cents ans d'histoire de la première congrégation mariale canadienne, mais d'examiner avec vous, remontant aux origines, la période des préparations et la période des débuts. Elles sont déjà très instructives.

\section{I. - LES PRÉPARATIONS}

\section{A. En France}

La Nouvelle-France, telle qu'elle fut et telle qu'elle demeure encore - Dieu merci - dans son fond chrétien, doit beaucoup à la Congréga- 
tion mariale française. A La Flèche, à Paris, à Caen, un peu partout en France, la Congrégation vivait, au XVII ${ }^{\circ}$ siècle, l'âge vigoureux de sa jeunesse. C'est dans les congrégations mariales que germaient les plus nobles desseins; c'est à la vertu solide et crucifiée des congréganistes que Dieu s'adressait pour les mener à bien. Notre pays en bénéficia.

Jérôme Le Royer de la Dauversière appartenait à la Grande Congrégation érigée au Collège royal de La Flèche, c'est-à-dire à la branche aînée de la Congrégation des Externes, laquelle comprenait aussi des citoyens, dits les Messieurs. Serez-vous surpris que Jérôme Le Royer ait eu, un 2 février, sa grande vision de 1630, en laquelle fut, en quelque sorte, conçue Ville-Marie, quand vous saurez que la Grande Congrégation de La Flèche avait pour titulaire la Purification de la sainte Vierge? Son prudent conseiller, en cette invraisemblable entreprise, devint, à partir de ce moment, le $\mathrm{P}$. François Chauveau, recteur du collège et directeur de la congrégation.

En 1642, le P. Alexandre de Rhodes, missionnaire au Tonkin, est de passage au célèbre collège parisien de Clermont. Le $P$. Jean Bagot l'invite à parler à ses congréganistes théologiens. Parmi les jeunes ecclésiastiques qui l'écoutent, il y a François de Montmorency-Laval, Ango de Maizerets, Henri Boudon, François Pallu, tous d'anciens élèves de La Flèche, passés à Paris pour leurs études théologiques. C'est la rencontre du P. de Rhodes avec les fervents congréganistes du P. Bagot qui, par les douces menées de la Providence, donnera à Québec, seize ans plus tard, son premier évêque.

La spiritualité de nos missionnaires des deux sexes avait été imprégnée, dès leur enfance, de la piété mariale des congrégations. Cinq au moins de nos Martyrs Canadiens étaient congréganistes et saint Charles Garnier, le seul dont nous connaissions la vie collégiale, se révèle, dans les notes que nous a laissées son frère carme, un parfait modèle de l'observation des règles. $\mathrm{Si}$ la bienheureuse Marguerite Bourgeoys n'appartenait pas à un groupe relevant de la Prima Primaria - celle-ci n'agrégeant pas encore de femmes - sa "Congrégation de Notre-Dame * en descend toutefois en ligne directe, par saint Pierre Fourier, congréganiste et fondateur, avec la bienheureuse Alix Le Clerc, des religieuses dites "Congrégantines ", qui s'établirent à Troyes en 1628 et à l'apostolat desquelles Marguerite Bourgeoys collabora, comme institutrice séculière.

Parmi les auxiliaires européens dont le P. Le Jeune, dans ses Relations enflammées, réclamait l'aide spirituelle et même matérielle, on trouve souvent mentionnées des congrégations mariales d'écoliers ou d'adultes. La réédition avignonnaise, en un seul volume, des Relations de 1634 et de 1635, sortie des presses de Jacques Bramereau en 1636, était précédée d'une longue lettre de l'imprimeur \& $A$ Messieurs les Préfet, Assistants, Conseillers et Confrères de la grande Congrégation de Notre-Dame, érigée au Collège d'Avignon, sous le titre de l'Immaculée. Conception de la Vierge». Jacques Bramereau, « imprimeur de Sa 
$+$

Admodng $R$ ringard, Patk

173

Pax christi

Clustas 5 abomus gantias Qustere Patronitati

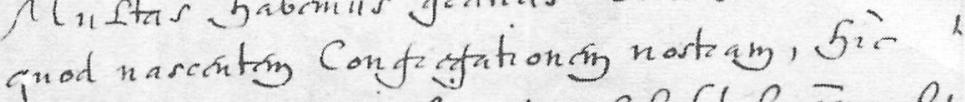

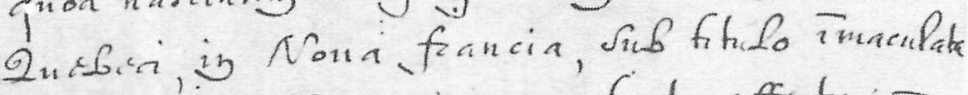
Concertiones B.2. Marie, fauto werthe-

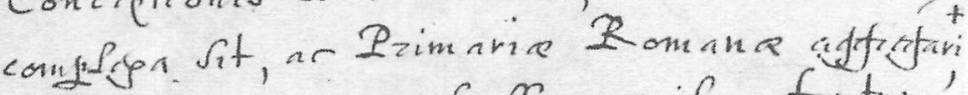
Mafrificas insupk buMas, quibus fantum binficing onnibus notum firect, Summisent. Prizamur ilfam B. nation Da, ut Lost-

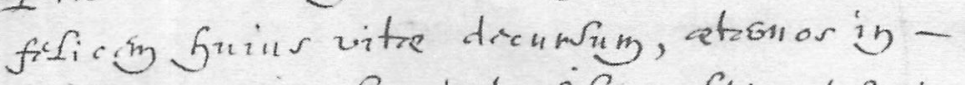

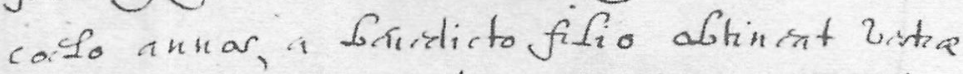
Patoonitati, cuins ofing Sanctioginis doteribus of Saruzicijs nos inige commondamus

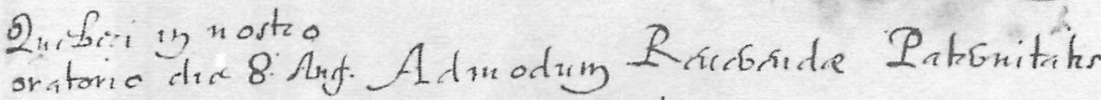
an. 1658 vostere

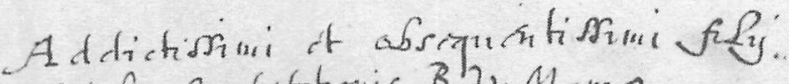

$$
\begin{aligned}
& \text { Sodalo Congrafationis B. U. Marie }
\end{aligned}
$$

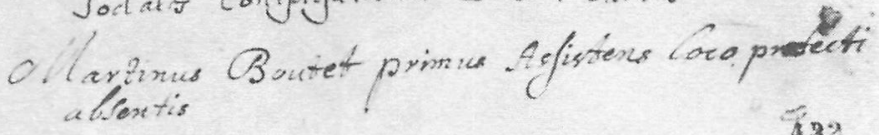

$$
\begin{aligned}
& 432
\end{aligned}
$$

La lettre des Congréganistes de Québec au T.R.P. Goswin Nickel (8 août 1658)

Ecriture du P. Claude Pijart - Signature de Martin Boutet 


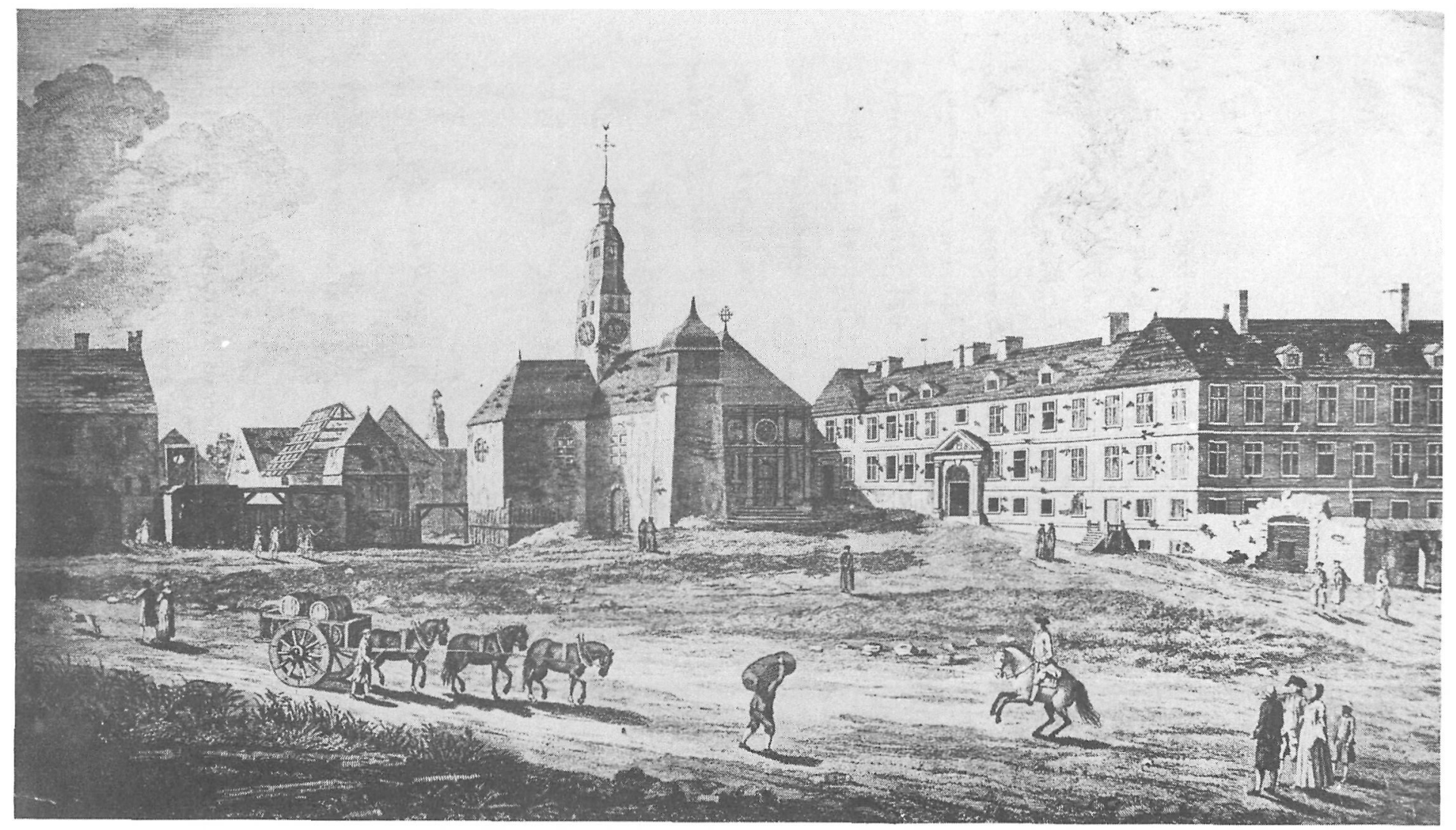

L'église et le collège des Jésuites, à Québec, en 1760 sur le site de l'hôtel de ville actuel

La chapelle de la Congrégation des Messieurs, au premier étage du collège, se distingue par ses fenêtres ouvertes, à gauche de la porte d'entrée 


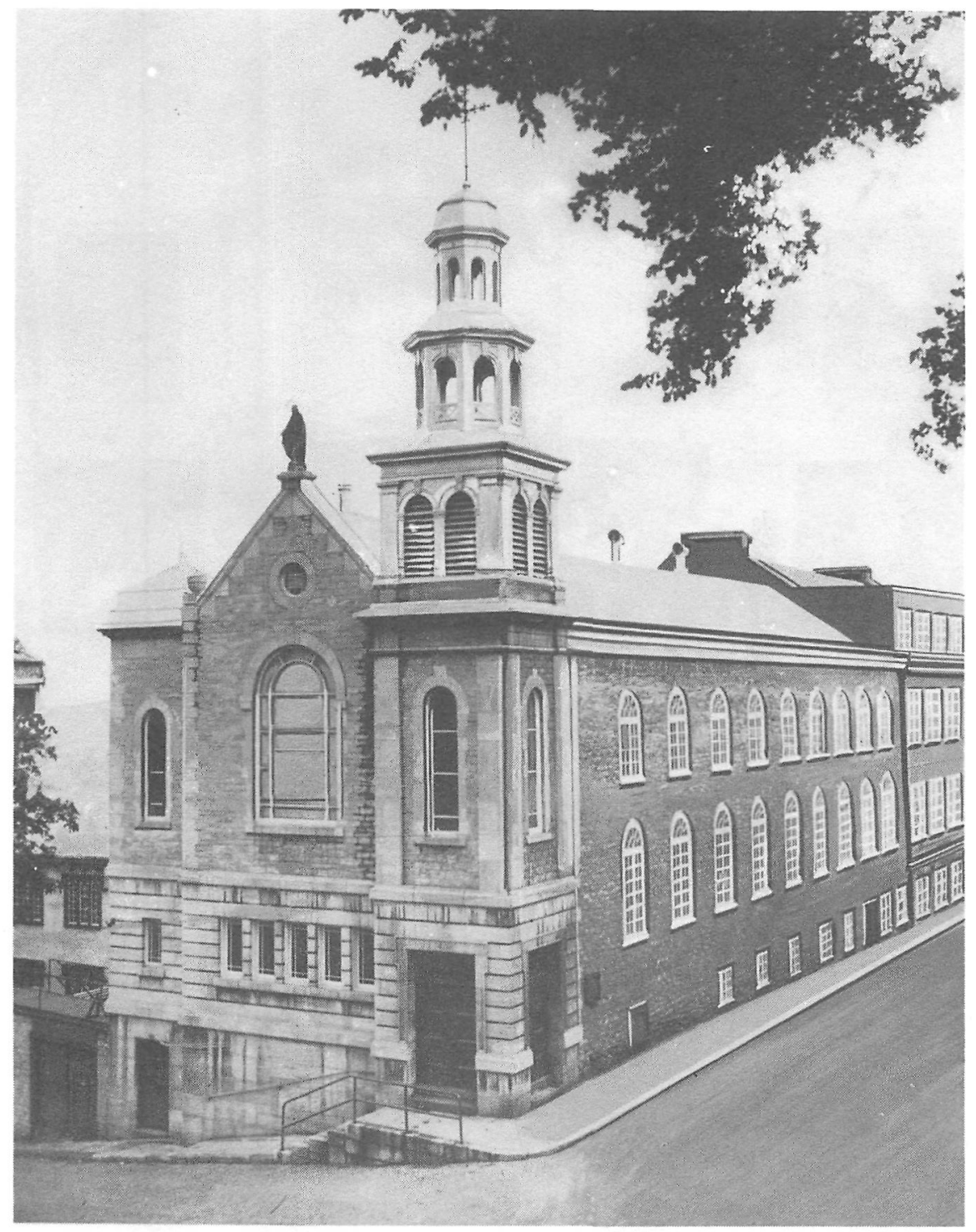

La chapelle de la Congrégation de la Haute-Ville au coin des rues d'Auteuil et Dauphine

Bâtie en 1817, sur un terrain donné par la Couronne, et transformée en 1930 


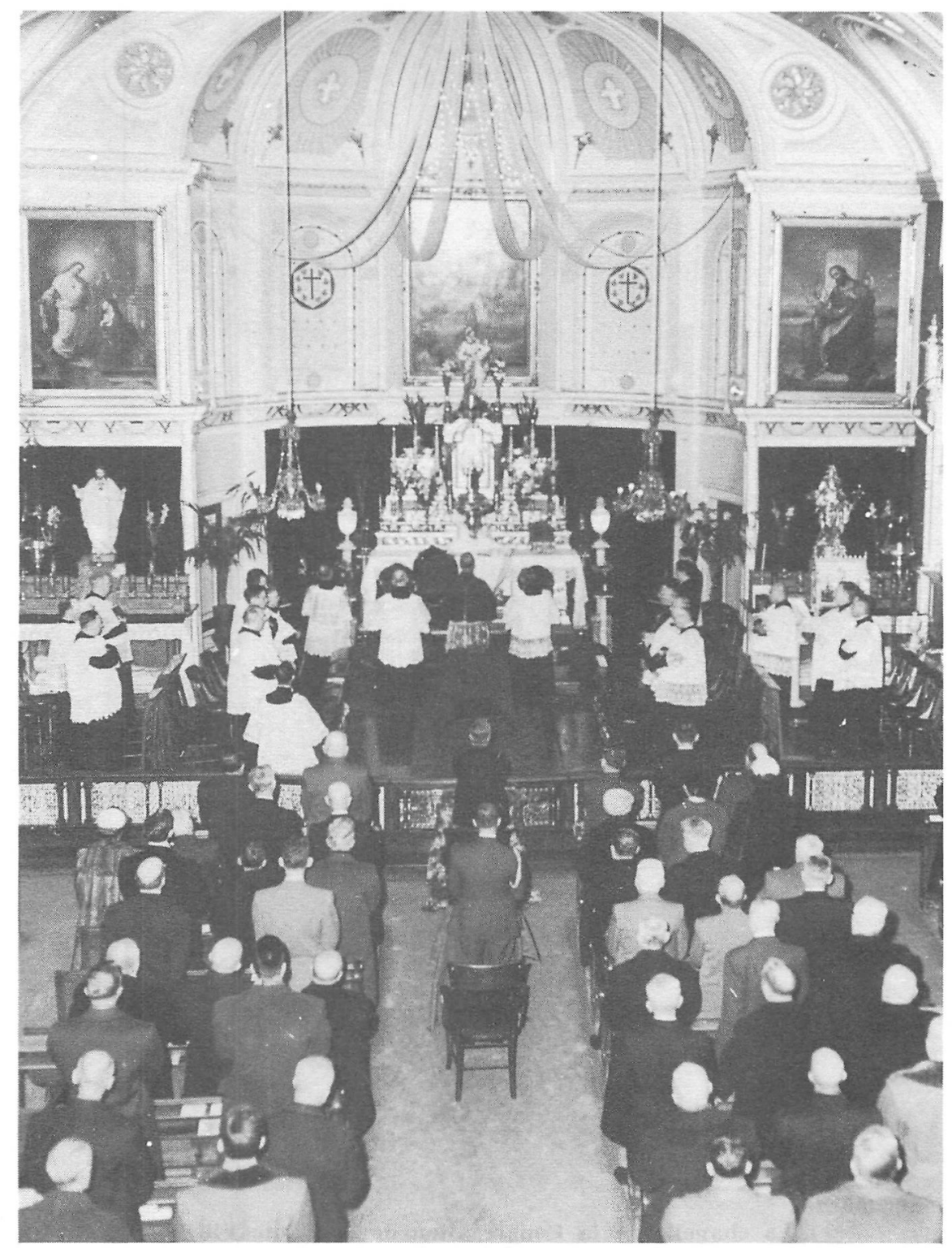

L'intérieur de la chapelle de la Congrégation jubilaire au matin du 3 février $195^{\text {t }}$

A l'autel, S.E. M Mrr l'Archevêque; au prie-Dieu, S.E. le Lieutenant-Gouverneur 
Sainteté, de la Ville et Université », était probablement congréganiste lui-même. Il exprimait ainsi sa piété et son zèle :

... Je vous présente cet ouvrage comme une pièce qui est toute acquise à votre Maîtresse et que j'appends comme un anathème [en bonne part, cétait un don] voué à cet auguste Oratoire où la Reine du Ciel voit autour de ses autels l'une des plus augustes assemblées qui soient en France... Que si vous agréez, Messieurs, cette offre, je redoublerai mes voux et mes prières pour hâter la conversion de ces peuples, afin que ma presse sue plus souvent et travaille sur quelque ouvrage plus grand, où vous reconnaîtrez que je suis, Messieurs, votre très humble et très obéissant serviteur.

Jacques BramereAd.

\section{B. Au Canada}

On possède à la Prima Primaria, parmi les documents de provenance canadienne, deux lettres latines de Charles-Pierre Le Gardeur, datées d'octobre 1652 et d'octobre 1653 - c'était le mois des derniers envois du courrier; elles encadrent une réponse, également en latin, du 9 février 1653, signée par le préfet et le secrétaire de la congrégation. mère. En termes chaleureux de part et d'autre, le requérant avait sollicité et on lui avait accordé la faveur assez exceptionnelle d'être inscrit dans la congrégation romaine, puisque le petit nombre des colons français et leur éparpillement empêchaient l'établissement d'une congrégation en Nouvelle-France.

Charles-Pierre Le Gardeur de Tilly avait évidemment de l'étoffe. Né en Normandie vers 1615, pourvu d'une culture humaniste que révèlent ses lettres, il avait environ vingt ans lorsqu'il arriva à Québec, le 11 juin 1636, en compagnie de sa mère veuve, de son frère et de sa søur, sur le navire qui emmenait au Canada le gouverneur Charles Huault de Montmagny. Navigateur comme son frère (l'amiral de Repentigny), gouverneur des Trois-Rivières de 1648 à 1650, membre du Conseil souverain dès la fondation de cet organisme et durant trentedeux ans consécutifs, deux fois député des Habitants à Paris, gouverneur militaire de Québec durant une absence du comte de Frontenac, époux de Geneviève Juchereau de Maure, père de quinze enfants * fort bien élevés et au cœur noble ", disaient les dépêches des administrateurs, et dont sept, en 1689, étaient simultanément « dans le service ", Charles Le Gardeur, qui, même à la fin de sa vie, "labourait la terre comme un vigneron ", mourut le 10 novembre 1695, âgé de soixante-dix-neuf ans, pauvre de biens mais riche de vertus. Dans le Journal des Jésuites, on le trouve portant le dais, à la procession de la Fête-Dieu, en 1650, partageant, comme convive, la joie de $P$. Chaumonot, le jour de la profession de son ami, peut-être son * confesseur ", sur le conseil duquel, dit-il, il a transmis à la Prima Primaria sa formule officielle de consécration à Marie et sollicité son inscription. La lettre de remerciement qu'il adressa, en 1653, à ses confrères et bienfaiteurs romains, résonne d'un tel accent de ferveur et de sincérité qu'on ne peut douter que Charles-Pierre Le Gardeur de Tilly ait vraiment préparé, parmi ses pairs, l'établissement de la première congrégation mariale canadienne. 


\section{Dans le coeur des missionnaires}

S'il n'y avait pas à Québec, en 1652, de congrégation mariale, comment expliquer que le registre de la Prima Primaria - registre recopié, certes, datant de 1785, après la suppression de la Compagnie de Jésus, mais registre authentique, basé sur les requêtes et concessions de lettres-patentes - comment expliquer, dis-je, que le registre de la Prima Primaria mentionne, à la date du 5 avril 1638, l'agrégation de trois congrégations canadiennes, en indiquant le titulaire de chacune : Notre-Dame de Lorette, les Epousailles de la bienheureuse Vierge Marie, Saint Joseph époux de bienheureuse Vierge Marie?

En principe, on ne peut canoniquement ériger et on ne peut canoniquement agréger qu'une congrégation existante. Par ailleurs, si ces trois congrégations canadiennes existaient vraiment en 1638, on ne comprend pas le silence du P. Le Jeune à leur endroit, tant dans ses Relations imprimées si complètes, que dans ses lettres si détaillées aux supérieurs de Paris et de Rome?

Je me suis permis d'émettre une opinion que l'on a d'abord qualifiée, au Secrétariat central de Rome, où sont les archives de la Prima Primaria, d'omnino reicienda - en bon français : d'inadmissible, mais que des lettres subséquentes de l'archiviste ont reconnue comme plausible. La voici.

Durant l'année académique 1637-1638, le P. Antoine Poncet de la Rivière était à parfaire ses études théologiques à Rome. Et il rêvait du Canada. Il l'avait connu, comme tout le monde, par les Relations, relations générales sur les missions de la Nouvelle-France, rédigées par le $\mathbf{P}$. Le Jeune, relations spéciales sur la mission huronne, rédigées par le P. de Brébeuf. Il avait été enthousiasmé par l'appel vibrant et réaliste que l'apôtre des Hurons avait lancé, en 1636, aux aspirants-missionnaires affamés de souffrances pour Jésus-Christ et les âmes. Il avait enseigné, durant sa régence, au petit Claude Martin et avait connu sa mère, devenue l'ursuline Marie de l'Incarnation, qu'il avait "conviée d'aller servir Dieu dans la Nouvelle-France ", lui adressant, en 1635, la Relation de l'année précédente. Lui-même y avait appris - comme y apprendrait sa correspondante - que le Canada avait pour patron principal saint Joseph. Et sa hantise du Canada, augmentant sa dévotion, l'avait poussé à obtenir du T.R.P. Général Mutio Vitelleschi la permission de substituer - ou du moins d'ajouter - à son nom d'Antoine celui de Joseph.

Son amour du Canada et sa dévotion à saint Joseph, Joseph-Antoine Poncet les avait insufflés à un jeune compatriote et compagnon du Collège romain, Pierre Chaumonot. Pour obtenir la grande faveur d'être envoyés en Nouvelle-France, tous deux firent ensemble le pèlerinage de Lorette et Pierre Chaumonot, enchérissant sur la piété de son ami, Joseph-Antoine, s'appela, lui, Pierre-Joseph-Marie Chaumonot. Au retour de Lorette, le T.R.P. Général accorda au jeune Chaumonot d'être ordonné prêtre, après deux ans seulement de théologie, et de partir pour le Canada avec le $P$. Poncet. Ils y arrivèrent tous deux le $1^{\text {er }}$ août 1639 , en même temps que Marie de l'Incarnation. 
Dans ces conjonctures, puisqu'il n'y avait certainement pas trois congrégations existantes au Canada en 1638, n'est-il pas vraisemblable que nos privilégiés aient obtenu de leur puissant protecteur - le T.R.P. Général des Jésuites, de qui relevaient les congrégations mariales - des agrégations en blanc, pour les groupements qu'ils rêvaient de fonder dans leur mission future, sur le modèle de la Prima Primaria, alors en plein essor?

Remarquez les titulaires : $B$. M. V. Lauretance, Desponsationis $B . M$. V., S. Joseph sponsi $B . M . V$. »; remarquez l'indication vague du lieu d'érection : "Canada, in America"; non pas telle ville, telle église, tel collège déterminés, comme c'est la coutume, mais « au Canada, en Amérique s. Et rappelez-vous - j'anticipe - le caractère entreprenant et franc-tireur du P. Poncet qui, durant son court passage, plus tard, à la cure de Québec, fondera coup sur coup, en douze mois, une confrérie du Scapulaire, une confrérie du Rosaire, une confrérie de Sainte-Anne et la Congrégation.

Le T.R.P. Mutio Vitelleschi aurait pu d'ailleurs invoquer un précédent : le 17 août 1595, le T.R.P. Claude Aquaviva avait fait remettre au P. Gilles de la Mata, procureur de la mission du Japon, des lettres patentes pour quatre congrégations qu'il l'autorisait à ériger et agréger sur place. Un précédent est toujours occasionné par une situation particulière : les deux fervents missionnaires en partance pour le lointain Canada avaient en mains, eux aussi, une bonne caụse.

Si les trois congrégations inscrites en 1638 n'existèrent jamais dans la réalité, ni chez les Français de Québec, ni chez les néophytes de Sillery, ni chez les chrétiens de la Huronie, il faut reconnaître cependant, à la gloire de la grâce divine, que la première congrégation à s'établir en terre nord-américaine fleurit chez les Hurons, lorsque ce peuple martyr se fut réfugié à la pointe de l'île d'Orléans. Or ce fut une réussite du P. Pierre-Joseph-Marie Chaumonot. Je n'ai pas à dire ici la ferveur de ces congréganistes, tant hommes que femmes, ni leurs fruits apostoliques, ni le témoignage du sang que rendirent à la foi plusieurs d'entre eux. Je signale seulement qu'elle fut fondée un an après la requête de Charles Le Gardeur à la Prima Primaria et que le P. Chaumonot, qui la fondait, était un des amis intimes de ce congréganiste obstiné. Pourquoi Charles Le Gardeur n'avait-il pas attendu cette fondation? Pour trois raisons, au moins : ce serait une congrégation de Hurons et non de Français; on ne savait pas comment elle réussirait; elle n'était pas encore agrégée - donc sans privilèges - et ne pourrait pas l'être, parce que mixte. Du reste, rien ne prouve qu'elle ait jamais été agrégée. Ce qui ne l'a pas empêchée de faire, à sa façon et hors cadres, beaucoup de bien, durant ses quelques années d'activité. Un des fruits de son établissement - qu'on ne trouve nulle part consigné dans les Relations ou le Journal, mais qu'on devine aisément, - ce fut de réveiller nos vieux chrétiens français, en train de se laisser dépasser par leurs convertis. 


\section{II. - LES DÉBUTS}

Et nous arrivons à 1657. Ouvrons, à cette année-là, le Journal. Janvier... Février... Le 14 février, dans la marge : « Douze congréga-

nistes. Première assemblée dans la chambre du Père Poncet. Au texte, qui est de l'écriture du supérieur de la mission et du collège, le P. Jean de Quen : "Le jour des Cendres, le P. Poncet fit la première assemblée, dans sa chambre, des Congréganistes de Notre-Dame. Ils étaient douze." Quelques lignes plus bas, au 24: "Monsieur Vignard dit la première messe dans la chapelle de la Congrégation de Notre-Dame, en la première assemblée des Congréganistes, où monsieur de Charny fut reconnu préfet de la dite Congrégation. »

C'est l'acte de naissance de notre Congrégation des Hommes de la Haute-Ville de Québec. Jamais, depuis lors, elle n'a cessé d'exister et de vivre; loin de là, elle a donné la vie à toutes les congrégations paroissiales masculines de Québec : une vingtaine. Privee de ses directeurs jésuites, à la suite de la conquête et de la suppression de la Compagnie de Jésus, menacée d'être chassée de son oratoire officiel, au vieux collège désaffecté, le curé, Mgr Plessis, pour plaider sa cause auprès du gouverneur Robert Shore Milnes, écrira, le 20 mars 1800 : a Elle fut d'abord composée de ce qu'il y avait de plus respectable parmi les gentilshommes et les bourgeois de la ville de Québec. On y compte encore aujourd'hui plus de deux cent cinquante membres, la plupart marchands, artisans, bons pères de famille ou jeunes gens remarquables par une conduite sans tache et sans reproche... Le maintien de cet institut est politiquement désirable pour la conservation non seulement de la religion et des mœurs, mais encore des principes de loyauté et d'obéissance qui doivent attacher les sujets au Roi et à son gouvernement. 》

\section{A. Premiers documents}

Mais revenons aux" débuts. Pour les étudier, les documents sont rares. Il y a bien, nous venons de le constater, le Journal des Jésuites, dans lequel les supérieurs du collège et de la mission consignaient, au jour le jour, les menus événements mémorables. Le Journal mentionne, ici et là, la Congrégation, la chapelle - ou l'oratoire - de la Congrégation (chapelle dès l'origine ou salle transformée en chapelle, à l'entrée du collège), où ont lieu, à l'occasion, les soutenances et les séances publiques; où sont inhumés tour à tour le Frère liégeois, le $\mathrm{P}$. de Quen, le $\mathrm{P}$. François du Peron; d'où partent les congréganistes pour aller faire leurs stations, le jeudi saint; où les femmes ne sont admises qu'aux grandes fêtes chômées. Mais le Journal - ce qui nous en reste du moins - ne dépasse pas 1668, c'est-à-dire les onze premières années de la Congrégation.

Et nos registres? Hélas! alors qu'on retrouve, au presbytère de Notre-Dame de Québec, les listes entières des confréries du Scapulaire, du Rosaire et de la Sainte-Famille, les premiers registres de la Congrégation, d'abord conservés au collège, siège de la Congrégation, puis, après le 
grand dérangement de la conquête et celui de la suppression de la Compagnie, confiés au secrétaire de la Congrégation, ont tous brûlé dans l'incendie des quartiers de Saint-Roch et de Saint-Jean-Baptiste, en 1845. Aussi nous faut-il chercher patiemment, partout où il semble possible d'en trouver, des bribes de renseignements.

Tous les ouvriers de l'histoire savent jusqu'à quel point ils sont redevables aux travaux de leurs devanciers. Pour ce qui regarde les documents de la Compagnie de Jésus (mère des congrégations mariales), nous devons une grande reconnaissance, nous Canadiens, aux PP. Martin, de Rochemonteix, Jones, Melançon, Desjardins, Léon Pouliot, Latourelle, Campeau et autres, qui nous ont transcrit, transmis ou interprété, avec grand mérite, les passages essentiels des archives romaines. Mais voici que la méthode moderne des copies photostatées ou microfilmées permet à tous les intéressés de lire eux-mêmes, dans le texte et à la lumière du contexte, avec le mérite non négligeable du déchiffrage et de la traduction, des cahiers complets de lettres et de mémoires. C'est ainsi que, l'an dernier, notre résidence de la rue Dauphine, à Québec, à laquelle sont aujourd'hui rattachés la Congrégation des Hommes de la Haute-Ville et le Secrétariat régional des Congrégations, pouvait obtenir, pour recherches historiques, le microfilm des lettres adressées, de 1611 à 1762, par les missionnaires jésuites de la Nouvelle-France au T.R.P. Général de la Compagnie de Jésus (Gallia 109-110) et une partie - la seule qu'on possède actuellement, à Rome - des réponses du T.R.P. Général (Gallia 39). Avec un bon compagnon de travail, le $P$. Joseph-Octave Bégin, qui prépare une étude exhaustive sur le $P$. Jérôme Lalemant, $j$ 'ai pu y retracer à peu près tout ce qui a trait aux congrégations canadiennes et compléter les renseignements extrêmement ténus du Journal et des Relations. Grâce à ces textes inédits et de première main, nous pouvons saisir sur le vif la vie concrète de la première congrégation mariale canadienne.

On me permettra de citer les textes dans leur langue originale, d'abord par souci d'objectivité, puis pour montrer à quelle école de haut humanisme fut formée notre race.

\section{B. L'AGRÉGaTion}

\section{LES PREMIERS CONGRÉGANISTES}

Suivant l'ordre chronologique, le premier document qu'on trouve, sur la Congrégation, dans la liasse Gallia 109, c'est une lettre adressée de Québec au T.R.P. Goswin Nickel et datée du 8 août 1658. La voici :

Admodum Reverende Pater

Pax Christi

Multas habemus gratias Vestræ Paternitati quod nascentem Congregationem nostram, hic, Quebeci, in Nova Francia, sub titulo immaculatæ Conceptionis B. V. Marix, tanto affectu complexa sit, ac Primariæ Romanæ aggregarit, magnificas insuper bullas, quibus tantum beneficium omnibus notum fieret, summiserit. Precamur illam B. matrem Dei, ut 
post felicem huius vitæ decursum, æternos in cœlo annos, a benedicto filio obtineat Vestra Paternitati, cuius etiam Sanctissimis precibus et Sacrificiis nos enixe commendamus.

Quebeci in nostro oratorio die 8 Aug. an. 1658

Admodum Reverendæ Paternitatis vestræ Addictissimi et obsequentissimi filii Sodales Congregationis B. V. Mariæ

Martinus Boutet primus Assistens loco præiecti absentis

Cette lettre confirme d'abord ce que nous savions : la Congrégation, fondée le 14 février a été agrégée à la Prima Primaria le 20 décembre suivant, sous le titre de l'Immaculée-Conception, cher aux missionnaires canadiens qui, en conformité avec leur vœu de 1635, avaient dédié à ce privilège de Marie les églises de Québec et de Trois-Rivières. Aujourd'hui, la congrégation tricentenaire honore la Purification de la sainte Vierge. Le changement date de 1836, alors que l'installation dans une autre chapelle, construite en 1817 et dédiée à la Purification, parut exiger une nouvelle agrégation. Qu'est devenu le beau diplôme magnificas bullas - reçu en 1658 ? S'il n'a pas brûlé en 1845, qui sait si on ne le retrouvera pas un jour dans quelque coin du grenier de la résidence de Québec... ou de l'archevêché, puisqu'en 1836 le directeur de la Congrégation - les Jésuites n'étaient pas encore revenus à Québec, ni même au Canada - était l'abbé Charles-Félix Cazeau, secrétaire de l'archevêque. L'imagination a son rôle à jouer, en histoire... Ses intuitions ne sont pas toujours folles.

La lettre du 8 août 1658 nous apprend aussi du neuf : le nom du premier assistant de la congrégation naissante, Martin Boutet. Traversé durant l'été de 1643, Martin Boutet de Saint-Martin, soldat, tailleur et menuisier lors de son engagement, devint petit à petit l'homme de science de la colonie, le professeur de mathématiques et d'hydrographie au Collège des Jésuites, l'ajusteur officiel des boussoles et des instruments d'arpentage. C'était, par surcroît, un excellent chrétien. Arrivé veuf en Nouvelle-France et père de deux filles, dont la cadette, Marie, prendra l'habit d'ursuline, en 1659, il s'était «donné " lui-même aux missionnaires. C'était un homme pieux, à qui le P. Lalemant offrait en étrennes des livres spirituels, par exemple, en 1647, L'exercice du chrétien. C'était enfin un homme d'œuvres, qui payait de sa personne : violoniste, il joue aux messes solennelles; maître-chantre, il défraie, en 1650 , le coût des manuels; deux ans plus tard, il prend charge, sous contrat, de l'entretien de l'église, de la formation des servants de messe et de l'organisation d'une petite-maîtrise. Ce doyen de nos a universitaires » canadiens était parfaitement chez lui dans la Congrégation, parmi les douze généreux du 14 février 1657 . Il méritait bien aussi la confiance de ses confrères, qui l'avaient choisi comme premier assistant.

Si Martin Boutet avait signé la lettre de remerciement des congréganistes au T.R.P. Général, c'était comme substitut du préfet absent. Ce préfet absent, nous le savons par la mention qu'a faite le Journal de son élection, le 24, février, "en la première assemblée de la Congrégation ", c'est "Mr de Charni ». Faisons connaissance avec le personnage : il a de la valeur. 
Charles de Lauzon, sieur de Charny - plus brièvement Charles de Lauzon-Charny - était le troisième fils de Jean de Lauzon qui, après avoir présidé, dès 1627, le Conseil de la Compagnie des Cent-Associés, négocié avec succès, en 1631, la restitution du Canada à la France, cédé à la Société de Ville-Marie toute l'île de Montréal, qui lui appartenait, était devenu, en 1651, quatrième gouverneur de la colonie. C'est l'année suivante de cette promotion que Charles de Lauzon vint le rejoindre. Il avait vingt-trois ans. Son père, qui aimait les titres, le nomma grandmaître des eaux et forêts. Le 12 août 1652 , un mois et demi après son arrivée, Charles épousait Marie-Louise Giffard, fille du seigneur de Beauport, qui mourut le 30 octobre 1656, « après une vie très pure et très innocente ", dit le Journal, laissant à son époux une fillette de seize jours. Au mois de juin précédent, Jean de Lauzon, âgé de soixantedouze ans et gouverneur aussi malheureux qu'il avait été bon administrateur financier, avait décidé de rentrer en France et de confier la colonie à son fils, le sieur de Charny. Celui-ci se trouvait donc, au printemps de 1657, gouverneur intérimaire de la Nouvelle-France.

Ce titre n'aurait pas suff, par lui-même, pour justifier les Jésuites de proposer à Charles de Charny-Lauzon d'être l'une des pierres de base de la Congrégation, si le jeune gouverneur, déjà reconnu pour un homme de bien, n'avait, en sortant victorieux d'une épreuve qui en aurait fait sombrer plusieurs, donné la vraie mesure de sa vertu. La Congrégation fut pour cette âme délicate et généreuse le nid bienfaisant où s'épanouit une vocation nouvelle : sept mois plus tard, le 18 septembre $1657, M$. de Charny remettait l'administration de la colonie entre les mains d'un ancien gouverneur qui rentrait d'un voyage d'affaires en France, Louis d'Ailleboust, et passait lui-même outre-mer pour se préparer à la prêtrise.

C'était donc le motif de l'absence du préfet, le 8 août 1658 . Lorsque le nouvel ordonné reviendra, le 16 juin 1659 , il aura fait le voyage en compagnie du vicaire apostolique, Mgr de Laval, qui l'honorera de sa totale confiance, au point de le nommer d'abord official et chanoine, puis grand vicaire et supérieur de l'Hôtel-Dieu. Il occupa ces deux dernières charges jusqu'en 1671, date de son départ pour La Rochelle où, retiré au Collège des Jésuites, il décéda vers 1690 .

Pour préfet, le gouverneur de la Nouvelle-France; pour premier assistant, un homme de science, dont les dires faisaient loi dans le pays. Quels étaient les dix autres? Sûrement le congréganiste de la Prima Primaria, Charles-Pierre Le Gardeur de Tilly; très probablement deux des trois laïcs qui « en notre chapelle * (l'oratoire de la Congrégation), chantèrent les lamentations des Ténèbres, le mercredi saint 28 mars 1657: le beau-frère de Charles Le Gardeur et son associé commercial, JeanPaul Godefroy, marguillier et membre du conseil; son neveu, JeanBaptiste Le Gardeur de Repentigny, jeune militaire de vingt-cinq ans, qui deviendra l'un des notables du pays; Pierre Duquet, le futur notaire (mais ce n'était encore qu'un collégien de quatorze ans, dont le nom dans le Journal n'est pas précédé, comme les deux autres, du respectueux - Monsieur $\gg)$. On peut vraisemblablement ajouter l'arpenteur Jean 
Bourdon, arrivé au Canada en 1634, décédé en 1668, dont l'esprit profondément chrétien, souvent signalé dans les mémoires contemporains, trouverait sa démonstration suffisante dans la vocation religieuse de ses quatre filles (deux chez les Ursulines, deux chez les Hospitalières). Un esprit profondément chrétien, voilà ce que requérait l'appartenance à la Congrégation et surtout la fondation d'une congrégation.

Plutôt que de risquer de faire sourire en poursuivant nos conjectures, revenons au certain. Ce « M. Vignard » qui " dit la messe dans la chapelle de la Congrégation de Notre-Dame, en la première assemblée des congréganistes », le 24, février 1657, qui est-ce ? Guillaume Vignard ou Vignal avait déjà vingt ans de prêtrise lorsqu'il traversa en NouvelleFrance. Le Journal, qui mentionne son arrivée, le 13 septembre 1648, en compagnie de " M. de Tilly ", le désigne alors comme le "prêtre des Ursulines ». Le chapelinat régulier des communautés féminines étant exclu des ministères auxquels peuvent se livrer les Jésuites, ceux-ci avaient plaidé auprès des moniales québecoises pour qu'elles fissent venir de France des prêtres séculiers. L'abbé Vignal remplira, dix ans durant, auprès des Ursulines la charge que l'abbé Jean Le Sueur de SaintSauveur, puis l'abbé Jean Le Bey exercèrent auprès des Hospitalières. Lorsque M. l'abbé de Queylus s'installera à la cure de Québec, Guillaume Vignal sourira à son invitation de se faire, comme lui, sulpicien. Au nom du curé, il ira, le 13 mars 1658, bénir la place de l'église du Petit-Cap (Sainte-Anne-de-Beaupré), puis prendra la mer pour aller faire sa solitude à Issy. Au retour, à l'été de 1659, en compagnie des trois fondatrices de l'Hôtel-Dieu de Ville-Marie, il sera missionnaire à Montréal, desservi par les Sulpiciens. Pris par les Iroquois, sur l'Ile-àla-Pierre, le 25 octobre 1661, il fut scalpé, tué, rôti et mangé par eux, à la Prairie de la Madeleine, deux jours après. Voilà le prêtre à qui les congréganistes et leur directeur avaient offert de célébrer, dans leur oratoire, le 24 février 1657, "la première messe, en leur première assemblée, où M. de Charny fut reconnu le préfet de la dite Congrégation *.

\section{Premiers directeurs}

Nous avons fait connaissance avec le P. Joseph-Antoine Poncet; nous savons son zèle et sa piété; nous avons deviné que c'était un tempérament porté aux excès. De fait tout en admirant ses grandes qualités d'apôtre, ses supérieurs eurent toujours quelque difficulté à le brider et ses compagnons à l'endurer. Cela rappelle la réponse de saint Ignace au provincial d'Allemagne, saint Pierre Canisius, qui s'était plaint de recevoir un sujet moins apte : a Avec la viande, mon Père, il faut accepter les os. "Dans le corps mystique, l'Esprit-Saint utilise tout ce qu'il anime. Le P. Poncet, avec tous ses défauts, que compensait beaucoup de zèle et de dévouement, fut un instrument des auvres de Dieu.

Redescendu à Québec avec ses confrères, à l'été de 1650, lors de la ruine de la mission huronne, le P. Poncet servit d'abord de compagnon à son ancien supérieur là-haut, le P. Paul Ragueneau, devenu vice- 
supérieur de toute la mission canadienne, durant l'absence du P. Jérôme Lalemant. Il l'accompagne sur la côte de Beaupré, jusqu'à la ferme du cap Tourmente, puis, dans l'autre direction, à Sillery, au cap Rouge et sur la côte Sainte-Geneviève. Il prêche et entend les confessions. Le 24 décembre, c'est lui qui bénit la nouvelle église paroissiale, y dit, ce jour-là, la première messe, et, la nuit suivante, la messe de Noël. Au carême de 1651 , il assure la prédication à la paroisse, dont le P. Barthélemy Vimont est le curé. Le 5 avril, il est parmi les jésuites qui assistent aux funérailles de la Mère Marie de Saint-Joseph, l'une des trois fondatrices. Le 21 août, on apprend à Québec qu'il a été fait captif par une bande d'Iroquois, en même temps qu'un Français qui coupait ses blés, "un peu au-dessus de Sillery, où la charité l'avait porté », note son nouveau supérieur, qui le connaît bien pour avoir longtemps vécu avec lui en Huronie, le $\mathrm{P}$. Le Mercier. Il passera deux mois épuisants parmi les Iroquois, aura les mains mutilées et ne sera ramené chez les siens qu'après beaucoup de prières de part et d'autre. A son retour, trois coups de canon clameront sa délivrance. Mais la captivité aura, paraît-il, accentué le pessimisme et l'irascibilité de son caractère. Il devenait, pour la petite communauté missionnaire, un problème passablement grave.

Devenu supérieur à son tour, à l'été de 1656, le P. Jean de Quen, un bon papa qui trouvait toujours des solutions de bonne entente, pensa résoudre le problème en confiant au dévoué malade la charge accaparante et exaltante de curé de Québec. Et voilà comment, en douze mois seulement de ministère, tous les projets en marche - ou en panne se trouvèrent réalisés comme par enchantement : confrérie du Scapulaire, confrérie du Rosaire, confrérie de Sainte-Anne (pour les menuisiers), Congrégation mariale (pour les notables), sans parler des mille et un détails que comporte l'installation dans une église neuve. Les documents sont là, au presbytère de Notre-Dame, rédigés à la perfection par le P. Poncet, de sa petite écriture ronde (tout allait rondement !), et contresignés par le P. de Quen. L'un de ces documents - un acte du notaire Audouart, relatif à la confrérie du Rosaire - qualifie assez originalement le curé de « vénérable et scientifique personne ». C'était évidemment tout un personnage, dont les franches coudées n'agréaient pas à tous. Le $\mathrm{P}$. de Quen, à qui arrivaient les plaintes, dut tenir compte de celles-ci et le retirer.

De fait, la lettre que les congréganistes adressaient au T.R.P. Goswin Nickel, le 8 août 1658, indique le changement; il n'apparaît pas dans le texte imprimé, mais il crève les yeux dans le texte original et sa photographie. C'est Martin Boutet qui signe les remerciements, mais ce n'est pas lui qui les a rédigés. Ce n'est pas le $\mathrm{P}$. Poncet non plus; c'est le P. Claude Pijart, ancien missionnaire de la Huronie et, ces dernières années, curé de Ville-Marie. Le changement, préparé, semblait-il, par la Providence, n'en avait pas moins occasionné toute une histoire.

Le 29 juillet précédent, le navire Le Nantois, avant de mouiller devant Québec, avait laissé descendre chez René Maheu, à l'île d'Orléans, M. de Maisonneuve et quatre ecclésiastiques sulpiciens destinés à prendre 
la direction spirituelle de Montréal. A la tête de ces derniers, il y avait l'abbé Gabriel de Queylus, récemment proposé par la Société de Montréal comme évêque de la Nouvelle-France, mais dont la candidature avait échoué à cause des préférences des Jésuites pour l'abbé François de Laval. En compensation, revanche ou simplement en attendant, l'abbé de Queylus avait facilement obtenu de l'archevêque de Rouen - dont l'autorité était alors reconnue, à tort mais de fait - des lettres patentes de grand vicaire en Nouvelle-France. Il n'y était pas dit que les nouveaux pouvoirs annulaient ceux du P. de Quen, mais l'étendue de la juridiction accordée à l'abbé de Queylus permettait de le croire.

Ignorant tout de cette situation, le bon P. de Quen vient chercher à l'île d'Orléans les nouveaux ouvriers de l'Evangile, leur fait visiter l'église, le collège, Sillery, les communautés, en somme leur fait les honneurs de son domaine. La journée se termine en un long entretien entre les deux supérieurs, sur l'organisation religieuse actuelle du pays et sur les modifications qu'entraînera l'installation des Sulpiciens à Montréal : le P. Claude Pijart, évidemment, reviendrait à Québec. En tout cas, le P. de Quen ayant, jusqu'à plus ample information, renoncé pour le moment à l'exercice de ses propres pouvoirs de grand vicaire, M. de Queylus exerça tout de suite les siens, en confirmant le statu quo de la cure de Québec, reconnaissant toutefois le droit, revendiqué par le supérieur des Jésuites, de déplacer à volonté ses sujets. Et l'abbé s'embarqua pour Ville-Marie.

Le retour prochain du P. Pijart parut-il au P. de Quen l'occasion la meilleure de remplacer le $P$. Poncet ? Celui-ci avait-il à la fin dépassé la mesure ? Le 28 août, six jours avant l'arrivée du P. Pijart, le pauvre curé est relevé de sa charge. Sur ses instances, le P. de Quen consent à l'envoyer dans la mission iroquoise d'Onontagué. Hélas! Ville-Marie était sur la route d'Onontagué et le grand vicaire résidait à Ville-Marie ! Il ne semble pas que le P. Poncet ait "monté " ce dernier contre son supérieur. Mais l'abbé de Queylus, apprenant la déposition du P. Poncet sans l'autorisation du grand vicaire, se fâcha, força le $P$. Poncet à l'accompagner à Québec, où ils arrivèrent le 12 septembre, à huit heures du soir. Il aurait voulu, sans doute, rétablir le P. Poncet dans sa charge; mais devant un refus énergique du P. de Quen, qu'appuyaient fermement ses conseillers, l'abbé riposta en refusant à son tour le P. Claude Pijart et en s'installant lui-même curé de la paroisse. Il ne la quittera qu'en septembre 1658 , un an plus tard, quand le $P$. de Quen lui aura montré les lettres qu'il vient de recevoir de l'archevêque de Rouen, lequel déclare qu'il a deux grands vicaires au Canada, l'un pour Québec, l'autre pour Montréal. Quant au P. Poncet, six jours après son retour forcé à Québec, on le voit s'embarquer avec M. de Charny (son préfet de congrégation, aspirant à la prêtrise) non pas dans un canot à destination d'Onontagué, mais sur le vaisseau du capitaine Poulet en partance pour l'Europe. Et les prochaines lettres à l'adresse du T.R.P. Général supplieront celui-ci de ne jamais lui permettre de revenir en Nouvelle-France. Il mourra missionnaire à la Martinique en 1675 et les Jésuites de la Nouvelle-France, reconnaissant 
son zèle et sa sincérité, feront alors pour le repos de son âme les suffrages prescrits pour les défunts de la mission.

$\mathrm{La}$ vénérable Marie de l'Incarnation, qui connaissait le P. Poncet de longue date, lui écrivait en France, le 7 octobre 1669: Votre révérence a été en ces lieux-là [chez les Iroquois]; elle y a semé, et les autres recueillent les fruits de ses travaux. Je m'assure qu'elle n'en aura pas moins de mérite que si elle les moissonnait elle-même. Ses mains mutilées en reluiront dans l'éternité, aussi bien que les autres parties de son corps qui ont porté tant de meurtrissures, et que ses oreilles qui ont été remplies de tant d'injures et de brocards. J'en ai encore le sentiment, mon très cher Père, et je bénis Dieu qui vous a donné le moyen de le porter et de le glorifier en votre corps par vos souffrances... * Autrement dit, à Dieu seul le jugement. Nous ne voyons que l'extérieur et l'apparent; lui sonde les reins et les cœurs. Puisque nos œuvres nous suivront dans l'au-delà et qu'elles aussi témoigneront pour nous, la Congrégation, qui est l'œuvre du P. Poncet, lui rendra témoignage. Déjà, sur terre, elle donne à entendre que l'arbre était bon.

La direction de la Congrégation n'était pas attachée à la fonction de curé; par contre, érection, agrégation et direction des congrégations étaient le privilège exclusif de la Compagnie de Jésus. Mis de côté comme curé, le P. Claude Pijart demeura le directeur de la Congrégation des notables de Québec, érigée au Collège des Jésuites, où elle avait son oratoire. Il le restera jusqu'en 1665 , alors qu'il réservera ses soins à la Petite Congrégation (d'écoliers) qu'il venait de fonder pour assurer la relève. La carrière du $\mathrm{P}$. Pijart n'offre pas grand matière pour le roman d'aventure; mais c'est vraiment lui qui a donné à la Congrégation, aujourd'hui tricentenaire et modèle en son temps de toutes les autres au Canada, son esprit surnaturel authentique. Trois documents - je parle de ceux qui ne sont pas de lui - nous renseignent sur le timbre de son âme.

Le premier, c'est l'appréciation de sa personnalité, telle que l'ont exprimée ses supérieurs au moment de l'admettre soit aux ordres sacrés soit à la profession. Ils disent :

Vires firmæ, ingenium et judicium bona, prudentia religiosa magna, experientia mediocris [pas encore beaucoup d'expérience], profectus bonus in theologia, melancholicus, talentum ad gubernandum, ad concionandum, ad res spirituales, ad missiones.

Pour quelqu'un qui est appelé à la vie religieuse et au sacerdoce, c'est prometteur.

Le second, c'est le jugement que portait sur lui, en 1655 , le supérieur de la mission canadienne, un vétéran, le $P$. François Le Mercier. Son triennat canonique étant à la veille de se terminer, le $\mathrm{P}$. Le Mercier suggère au T.R.P. Général le nom du P. Pijart pour le remplacer :

Porro de superiore in meum locum anno proximo substituendo, significavi iam Paternitati Vestræ, superioribus meis litteris, mentem meam : nimirum existimare me optime his missionibus consultum iri, si P. Claudium Pijart nobis præficiendum curet, propter eximias eius virtutes : pietatem, mansuetudinem, humilitatem, caritatem, nostrarum 
constitutionum intelligentiam, prudentiam non politicam sed religiosam et sanctam, etc. de quibus fusius scripsi Patri Provinciali, ex ipsiusmet mandato.

Dans cette édifiante liste de vertus religieuses, on aura remarqué l'insistance du P. Le Mercier à signaler au T.R.P. Général la qualité supérieure de la prudence de son candidat : une prudence non pas naturelle et mondaine mais surnaturelle et sainte. D'autres correspondants ex officio du T.R.P. Général avaient sans doute proposé d'autres noms : le T.R.P. Nickel désigna, en 1656, le P. Jean de Quen. Mais cette préférence n'enlevait pas au P. Pijart ses vertus et ses dons.

Le P. Pijart mourut à Québec à l'âge de quatre-vingt-trois ans, le 16 novembre 1683. Ce jour-là même, le $P$. Thierry Beschefer, son supérieur, commençait de la façon suivante la lettre qu'il adressait au P. Charles Pijart, religieux minime, à Paris : «J'écris à Votre Révérence pour lui mander la mort du $\mathbf{P}$. Claude, dont les rares vertus font dire à ceux qui l'ont connu que cette mort a donné au ciel un nouveau saint en sa personne.» Deux extraits de la notice nécrologique - ou Abrégé de sa vie - que rédigea le P. Martin Bouvart démontreront le bien-fondé dé cette affirmation générale et mettront en pleine lumière la belle figure du second directeur de la Congrégation.

B'abord la promesse des fleurs : "Aux collèges de La Flèche et de Paris, il a été l'exemple des congréganistes, des pensionnaires et de tous les écoliers par sa modestie angélique et par son assiduité continue à la prière et à l'étude. »

Puis la richesse des fruits : "Il n'est pas croyable quel bien il a fait dans la ville de Québec et aux environs, en s'acquittant parfaitement des emplois de missionnaire, de curé [il le fut sept ans à Ville-Marie], de prédicateur, de catéchiste, de confesseur, de Père [sic] de la Congrégation, de visiteur des malades à l'hôpital, des criminels dans les prisons, des soldats dans leur corps de garde et des ouvriers dans leurs ateliers. Il instruisait, avec un zèle infatigable, toutes sortes de personnes, dans tous ces lieux différents. De plus, il enseigna, dans notre collège, tantôt les humanités ou la rhétorique, tantôt la philosophie, les cas de conscience [la morale] et la théologie. Ajoutez encore plusieurs offices de la religion [i.e. de la vie religieuse] et plusieurs charges qu'il a eues, car il était propre à tout sans exception.»

Voilà l'homme que la Providence, en le soustrayant au supériorat de toute la mission, réservait à la Congrégation naissante pour lui insuffler un esprit, le véritable esprit de la Congrégation. "Mementote prapositorum vestrorum " (Hebr., XIII, 7). Souvenons-nous de nos " fondateurs ", qui nous ont fait entendre la parole de Dieu et, considérant l'issue de leur carrière, imitons leur foi.

\section{La FERVEUR DES PRÉMICES}

Qu'on nous permette encore d'offrir l'eau fraîche et pure des sources, en extrayant de la correspondance annuelle qui s'échange entre Québec et Rome les courts mais significatifs passages racontant les 
progrès de la Congrégation. A ces sources primitives personne avant vous, que je sache, ne s'est abreuvé.

a) Lettre du P. Pijart au T.R.P. Général, le 26 août 1658 :

Gratias ago Paternitati Vestræ quod nascentem Congregationem cuius mihi cura commissa est, sub titulo immaculatæ Conceptionis Dei Genetricis Mariæ, tanto affectu complexa sit [on reconnaît l'auteur du message des Congréganistes], magnificisque bullis munierit ac exornarit. Crescit illa in dies et numero et merito...

\section{b) Réponse du T.R.P. Général, le 16 décembre 1658 :}

Recrearunt me literæ $R$. Væ 26 Augusti datæ, ex quibus intellexi felicia crescere in dies Congregationis initia, quæ, cum industriæ et vigilantiæ Reverentiæ Vestræ debeantur, ita ea ipsi gratulor...

c) Lettre du P. Pijart au T.R.P. Général, le 31 septembre 1659, trois mois et demi après l'arrivée de Mgr de Laval :

Gratias habeo maximas Vestræ Paternitati quod de Congregatione B. V. hic, inter barbaros, erecta, talem sensum et tantam curam habeat. Crescit in dies et numero et merito, præsertim ex quo Illustrissimus D. Episcopus Petrex, huc ad nos a Sanctitate sua directus, palam se sodalem professus, eam cepit colere, missam interdum in Sodalitio celebrare, cum sodalibus nosocomio inservire...

Mgr de Laval, on le sait, était congréganiste depuis longtemps.

d) Le T.R.P. Général répond, le 2 février 1660 :

Mirifice lætor quod, uti ex literis $R$. Væ 31 Sept. proxime lapsi ad me datis intelligo, Beatæ Virginis Sodalitas, cuius ipsa curam suscepit, in numero et merito augeatur in dies. Sodalium pietati haud dubio prodibit exemplum illustrissimi Episcopi Petreensis, qui mare trajicere voluit renascentem istam Ecclesiam crucibus abundantem gubernare...

e) En 1660 et 1662, deux brèves mentions optimistes, dans les lettres du P. Pijart :

Sodalitas B. V. Mariæ crescit in dies.

Congregatio B. V. floret.

f) Le P. Henri Nouvel, quatrième directeur de la Congrégation, indique au T.R.P. Général, le 31 août 1670 , la consolante constatation qu'il a faite :

Perseverat inter nos [les Jésuites] et Dominum Episcopum [Mgr de Laval] eiusque sacerdotes [les prêtres du Séminaire] summus animorum consensus; ea est eorum omnium pietas ut Sodalitium Beatissimæ Virginis apud nos erectum frequentent, singulisque diebus Sabbati meditationum explicationi intersint...

Il y avait donc, chaque samedi, à l'oratoire de la Congrégation, la réunion hebdomadaire, durant laquelle on s'initiait à la méditation, et les prêtres du séminaire venaient au collège se joindre aux laïcs congréganistes pour la réunion. Les élèves du petit séminaire, qui suivaient les cours du collège, firent de même puisque, pour les deux institutions, il n'y eut jusqu'à 1768 qu'une seule congrégation d'écoliers, celle qu'avait commencée le $P$. Pijart en 1664 et qui serait agrégée le 7 février 167.2. 
g) Le P. Jérôme Lalemant, plusieurs fois supérieur de la mission et saint religieux, s'arrêtait chaque jour dans l'oratoire de la Congrégation, pour y prier la sainte Vierge. Son éloge nécrologique le signale, en 1673 : a Fuit (egrege pius) et in Beatam Virginem; singulisque diebus in Sodalitium ibat, ut eam salutaret. »

h) Un autre supérieur des plus remarquables en Nouvelle-France, le P. Claude Dablon, explique dans quelles conditions méritoires de personnel s'accomplit le bien qui se fait au collège. Cette lettre, au T.R.P. Oliva, est du $1^{\text {er }}$ septembre 1679.

De iis vero quæ fiunt in hoc collegio Quebecensi, nihil dico. Vix credi potest quanta et a quam paucis peragantur. Enimvero ad conciones in nostro templo singulis diebus dominicis habendas, matutinis horis et serotinis; ad christianam doctrinam tradendam; ad conciones item in Ecclesia cathedrali et in aliis hujusce urbis templis; ad audiendas confessiones, ad nos enim frequenter Gallorum prope omnium concursus; ad duas congregationes regendas, alteram scholasticorum [i.e. discipulorum], alteram civium et artificum, hanc autem numero plus quam 300 sodalium, quorum nonnulli in oppidis remotis siti ad omnia sodalitii exercitia conveniunt; ad exhortationes in xenodochio bis aut ter in hebdomada ad ægrotos; item ut necesse est ad incarceratos; denique ad Rhetoricam et inferiores scholas; ad hæc, inquam, et alia permulta, tres hic habeo tantum de Nostris, P. T. Beschefer, P. M. Bouvart, et Magistrum [le régent scolastique, chargé de la Petite Congrégation] Claudium Touvenot. Alii tres Patres laboribus et æatate fracti sunt : P. Cl. Pijart octogenarius, P. Andreas Richard item octogenarius et P. Petrus Chastelain septuagenario major.

Il y a beaucoup de leçons à retirer de ce tableau : pour nous, prêtres, énergie au travail, concentration sur les besognes spirituelles, dévouement aux âmes de toutes les catégories; pour les congréganistes, fidélité, coûte que coûte, aux réunions hebdomadaires, ardeur au recrutement : ils étaient trois cents, en 1679, dans la toute jeune région de Québec.

Le $P$. Dablon, qui nous fournit ces renseignements, connaît bien le groupe - la Congrégation des Citoyens, ou la Grande Congrégation, comme on la désigne alors, pour la distinguer de la Petite Congrégation des écoliers. C'est lui qui a succédé au P. Pijart, en 1665, lorsque celui-ci fut chargé des jeunes; après le bref séjour du P. Nouvel à Québec (1669-1670), il l'a reprise en mains et en conservera la direction jusqu'en 1693, ce qui, depuis 1665, donne un total de vingt-sept ans. Lorsque, à la fin d'un long et fécond supériorat (1670-1680; 1686-1693), il aura réussi l'établissement, à Ville-Marie, d'une église des Jésuites, d'une résidence et d'un embryon de collège, il ne manquera pas de favoriser l'érection d'une congrégation mariale parmi les Montréalais : Bonum est diffusivum sui. La lettre du $1^{\text {er }}$ octobre 1693, par laquelle le P. Dablon remercie le T.R.P. Thyrse Gonzalès, est encore inédite. Le passage suivant intéressera nos belles congrégations du diocèse de Montréal et particulièrement celle de la paroisse de Notre-Dame :

Multum autem debemus paternitati Vestræ quod aggregationem Sodalitii civium recens instituti in urbe Mariana Sodalitio Romano tam liberaliter concesserit quodque Instrumentum [le diplôme] ea die confici jusserit ad nos prima data occasione transmittendum.

La nouvelle congrégation reçut pour titulaire l'Assomption. 


\section{CONCLUSION}

Le plan miséricordieux de la Providence sur les origines religieuses du Canada a donc réservé aux congrégations mariales un rôle important dans cette "épopée mystique ".

Au cour de la Vieille-France, elles ont contribué à former et à soutenir un grand nombre des hommes et des femmes, clercs et laïcs, dont Dieu voulait se servir pour jeter chez nous les bases de son Royaume : Jérôme Le Royer de la Dauversière, les Martyrs canadiens et leurs émules jésuites, François de Montmorency-Laval et quelques-uns de ses prêtres séculiers, Marguerite Bourgeoys et ses compagnes, les Le Gardeur, les Lauzon et d'autres. Dans la Nouvelle-France, la Congrégation a groupé l'élite à la fois humaine et chrétienne de la colonie naissante : élite peu nombreuse mais réelle, tirée de l'administration, de la science, du professorat, du commerce, de l'agriculture, de l'artisanat, du clergé. Sous la bannière de l'immaculée Vierge Marie, à l'école de Celle qui a réalisé parfaitement les visées de Dieu sur l'humanité, ces humbles citoyens de la terre, avec leurs tempéraments imparfaits, leurs ambitions politiques ou commerciales, leurs problèmes personnels et familiaux, ont accepté de livrer à fond le bon combat et de coopérer positivement à l'œuvre de l'Eglise dans leur jeune pays, c'est-à-dire au triomphe, en eux et autour d'eux, de la vertu sur le vice, de la grâce sur la nature, de Jésus-Christ sur Satan. C'est l'idéal même des congrégations, servantes de l'Eglise.

Nous espérons pouvoir raconter un jour l'œuvre subséquente accomplie par la Congrégation en terre canadienne. Pour le moment, constatons deux faits et tirons-en la conclusion qui s'impose.

Après la ferveur des prémices et l'efficacité de l'âge d'or, les congrégations mariales - instruments de la grâce entre des mains d'hommes - ont subi, non seulement au Canada mais partout, pour diverses raisons, entre autres le manque d'adaptation et de mise au point, un ralentissement puis un assoupissement qui ont fini par faire douter de leur actualité, même de leur survie. Leurs assemblées. hebdomadaires, en particulier, devenues çà et là mensuelles, ont un peu partout dégénéré en une routine monotone de prières vocales, sans travail sur soi, sans effort d'apostolat, scandée, une fois l'an, d'élections et fournissant tout au plus aux membres en règle la distinction fort appréciée d'un honneur spécial au mariage ou aux funérailles.

Mais on ne doit jamais désespérer de la grâce. Malgré leur réelle inertie, brûlait au sein des congrégations la flamme discrète d'une sincère dévotion à la sainte Vierge, exprimée par la consécration totale de soi-même à l'Immaculée. Sur cette flamme le Saint-Père a soufflé. Par sa constitution apostolique Bis saculari, qu'il a qualifiée de « charte des congrégations mariales ", il a rappelé aux congréganistes de tous les pays la confiance qu'avait toujours témoignée à leur mouvement, depuis sa fondation, le Vicaire du Christ. Il les a fortement engagés à remonter aux sources, à s'inspirer des règles traditionnelles, à réveiller la ferveur 
d'antan, à retrouver les élans apostoliques d'autrefois, à reprendre leur place dans les rangs de l'Action catholique.

Plusieurs de nos congrégations canadiennes ont entendu cet appel à lá générosité. Le rayonnement de plus en plus extensif et intensif de nos deux secrétariats nationaux, l'organisation et la mise en marche de secrétariats : régionaux, l'élan remarquable des congrégations de jeunes en sont de consolantes preuves. Mais plusieurs ne suffit pas. Le troisième centenaire de la première congrégation mariale canadienne offre à toutes et à chacune des deux mille congrégations canadiennes agrégées à la Prima Primaria l'occasion exceptionnelle de prêter enfin l'oreille aux ordres du Saint-Père, de s'appliquer à elles-mêmes ses directives précises, de secouer - énergiquement et violemment, s'il le faut - leur routine, de ressusciter à la vraie vie et de redevenir, comme aụ grand siècle, une force dans l'armée du bien.

C'est, à n'en pas douter, ce que demandent pour nous, là-haut, cette année, les fondateurs et les bénéficiaires du mouvement lancé en 1657; c'est également l'idéal que nous proposait, le 26 janvier 1957, Son Fxcellence Mgr Joseph Gawlina, archevêque de Madytos et directeur de la Fédération mondiale des Congrégations mariales, dans sa lettre de félicitations à la Congrégation des Hommes de la Haute-Ville de Québec : - L'exemplaire fidélité de vos anciens invite les plus jeunes à persévérer dans un attachement toujours plus profond à Notre-Seigneur JésusChrist. La Sainte Vierge, votre modèle, est restée fidèle à son divin Fils de la crèche à la croix. Imitez-la et que cet anniversaire - selon les vœux et désirs de vos évêques - soit le point de départ d'une période encore plus fervente et plus féconde non seulement pour votre Congrégation jubilaire, mais aussi pour toutes les congrégations mariales canadiennes!»

\section{Adrien Pouliot, s.j.,}

directeur de la Congrégation des Hommes de la Haute-Ville et du Secrétariat régional des Congrégations mariales à Québec.

\section{BIBLIOGRAPHIE}

Outre les Relations des Jésuites et le Journal des Jésuites, les archives privées du Collège Sainte-Marie, à Montréal, de la résidence des Jésuites et de la Congrégation des Hommes, à Québec, celles du Séminaire de Québec, de la paroisse Notre-Dame et de l'archevêché, que mirent en pleine lumière les archives romaines de :la Compagnie de Jésus, signalons, comme sources de renseignements pour ce travail :

1. Acies Ordinata, revue du Secrétariat central des Congrégations mariales, à Rome. Entre autres documents, elle publie en appendice, depuis 1954, le contenu du plus ancien iegistre de la Prima Primaria.

2. Autobiographie du P. Chaumonot, de la Compagnie de Jésus (éd. Félix Martin, Paris, 1885).

3. Bulletin des Recherches historiques (Lévis), en particulier, au volume XLV, $\mathrm{n}^{\circ} 2$ (février 1939), pp. 33-42; "Le premier congréganiste canadien-français [sic] - Charles Le Gardeur de Tilly ", par le P. Emile Villaret, s.j. (du Secrétariat de Rome, auteur d'une histoire détaillée des Congrégations mariales [Beauchesne, Paris, 1946], dont il n'a pu compléter que la première partie : 1540.1773).

4. Dictionnaire général du Canada, par le R.P. L. Lejeune, o.m.i. (2 vol., Ottawa, 1931). 
5. Ecrits spirituels et historiques de Marie de lIncarnation (éd. Dom Jamet, Paris-Québec, 1930, complétée par l'édition Richaudeau, Paris-Leipzig, 1876).

6. Histoire de la Compagnie de Jésus en France, par le P. Henri Fouqueray, s.j. (5 vol., Paris, 1910-1925).

7. La vie ardente de saint Charles Garnier, par le P. Florian Larivière, s.j. (Montréal, 1957).

8. Le Collège Henri IV de La Flèche, par le P. Camille de Rochemonteix, s.j. (4) vol., Le Mans, 1889).

9. Les Jésuites en Nouvelle-France au XVII siècle, par le même (3 vol., Paris, 1895-1896).

10. L'Hôtel-Dieu, premier hôpital de Montréal, par Sœur Mondoux, r.h.s.j. (Montréal, 1942).

11. Lettres du Bas-Canada, publication privée des Jésuites canadiens de langue française.

12. Marguerite Bourgeoys, par Dom Albert Jamet, o.s.b. (2 vol., Montréal, 1942).

13. Un modèle pour les Congrégations mariales: la bienheureuse Marguerite Bourgeoys, par l'abbé Joseph Gariépy, p.m.é. (Secrétariat national des CC.MM., Montréal, 1954). 